Mathematical Modelling and Analysis

Volume 22 Number 4, July 2017, 503-513

https://doi.org/10.3846/13926292.2017.1329755

(c) Vilnius Gediminas Technical University, 2017
Publisher: Taylor\&Francis and VGTU

http://www.tandfonline.com/TMMA

ISSN: $1392-6292$

eISSN: 1648-3510

\title{
Fractional Order Barbalat's Lemma and Its Applications in the Stability of Fractional Order Nonlinear Systems
}

\author{
Fei Wang ${ }^{a}$ and Yongqing Yang ${ }^{a, b}$ \\ ${ }^{a}$ School of Internet of Things, Jiangnan University \\ 214122 Wuxi, China \\ ${ }^{b}$ School of Science, Jiangnan University \\ 214122 Wuxi, China \\ E-mail(corresp.): yongqingyang@163.com \\ E-mail: fei_9206@163.com
}

Received September 8, 2016; revised May 7, 2017; published online July 15, 2017

\begin{abstract}
This paper investigates fractional order Barbalat's lemma and its applications for the stability of fractional order nonlinear systems with Caputo fractional derivative at first. Then, based on the relationship between Caputo fractional derivative and Riemann-Liouville fractional derivative, fractional order Barbalat's lemma with Riemann-Liouville derivative is derived. Furthermore, according to these results, a set of new formulations of Lyapunov-like lemmas for fractional order nonlinear systems are established. Finally, an example is presented to verify the theoretical results in this paper.
\end{abstract}

Keywords: fractional order system, nonlinear differential equation, stability.

AMS Subject Classification: 93D05.

\section{Introduction}

Asymptotic stability analysis of non-autonomous systems is generally much harder than that of autonomous systems, since it is usually very difficult to build a Lyapunov function with a negative definite derivative. The well-known Barbalat's Lemma is a purely mathematical result concerning the asymptotic properties of functions and their derivatives, which has been a powerful tool to asymptotic analysis of the dynamic systems especially time-varying nonlinear systems [22]. Many publications generalized the integer order Barbalat's Lemma. By the generalised Barbalat's lemma, the uncertain complex dynamical network is proved to be locally or globally asymptotically synchronised [9]. 
Based on a set of new versions of Barbalat's lemma, Lyapunov-like lemma are established in [7]. Wu considered the extensions of Barbalat's lemma to the stochastic case to analyze stochastic stability [25]. More results about stability analysis for systems via Barbalat's Lemma can be seen in $[1,12,15]$ and their references. Fractional order systems have attracted many attentions due to their advantages in some fields such as electrochemistry [11], diffusion [20], viscoelastic materials [21], control [2,19], biological systems $[10,17,18]$ and so on. Stability has been widely investigated as an important performance index of control systems. Lots of results can be found about stability for the fractional order systems $[8,14,16]$. There are also some papers which dealt with the stability problem of fractional system via the integer order Barbalat's lemma. $\mathrm{Li}$ and Chen developed a novel adaptive fractional-order feedback controller to synchronize almost all familiar fractional-order chaotic systems [13]. In [27], adaptive sliding mode approach was applied to synchronize two different fractional-order chaotic systems, which the integer order Barbalat's Lemma was also used. Noting that the Barbalat's Lemma adopted in [13] and [27] is described by integer derivative and integral. More recently, based on some conditions upon its fractional integral, Barbalat-like lemmas for fractional order integrals have been used to conclude the convergence of a function to zero in [6]. There were also some results about boundedness and convergence on fractional order systems have been published in [5].

Inspired by the above-mentioned discussions, it's natural for us to ask a question: can the classical integer order Barbalat's lemma be generalized to the fractional order? [6] only partially answer this question. In which, the convergence of a function just related to its fractional integral, and the function has been studied in which must be bounded. In this paper, the relationship between uniform continuity of a function and the boundedness of its fractional derivative will be built, then a fractional order Barbalat's Lemma described by fractional derivative will be proposed. Both Caputo operator and RiemannLiouville operator will be discussed. According to above results, a set of new formulations of Lyapunov-like stability lemmas for fractional order nonlinear systems are established.

\section{Preliminaries}

In this section, some basic notions and properties for fractional calculus and fractional differential equations are recalled. For further details, one can refer to $[2]$.

DEFInItion 1. The fractional integral of order $\alpha$ for a function $x(t)$ is defined as

$$
D_{t_{0}, t}^{-\alpha} x(t)=\frac{1}{\Gamma(\alpha)} \int_{t_{0}}^{t}(t-\tau)^{\alpha-1} x(\tau) d \tau,
$$

where $\Gamma(\cdot)$ is the Gamma function, $t \geqslant t_{0}$ and $\alpha>0$.

There are different definitions for fractional-order derivatives. Among which Caputo derivative and Riemann-Liouville derivative are the most frequently concerned in research. The formula of them are defined as follow: 
Definition 2. The Caputo fractional derivative of function $x(t)$ is defined as

$$
{ }^{C} D_{t_{0}, t}^{\alpha} x(t)=\frac{1}{\Gamma(m-\alpha)} \int_{t_{0}}^{t}(t-\tau)^{m-\alpha-1} x^{(m)}(\tau) d \tau
$$

where $m-1<\alpha<m, m \in Z^{+}$.

Definition 3. The Riemann-Liouville derivative of fractional order $\alpha$ of function $x(t)$ is defined as

$$
{ }^{R L} D_{t_{0}, t}^{\alpha} x(t)=\frac{d^{m}}{d t^{m}}\left[D_{t_{0}, t}^{-(m-\alpha)} x(t)\right]=\frac{1}{\Gamma(m-\alpha)} \frac{d^{m}}{d t^{m}} \int_{t_{0}}^{t}(t-\tau)^{m-\alpha-1} x(\tau) d \tau
$$

in which $m-1<\alpha<m, m \in Z^{+}$.

In the following, it is always assumed that $0<\alpha<1$, then

$$
\begin{aligned}
& { }^{R L} D_{t_{0}, t}^{\alpha} x(t)=\frac{d}{d t}\left[D_{t_{0}, t}^{-(1-\alpha)} x(t)\right]=\frac{1}{\Gamma(1-\alpha)} \frac{d}{d t} \int_{t_{0}}^{t}(t-\tau)^{1-\alpha-1} x(\tau) d \tau, \\
& { }^{C} D_{t_{0}, t}^{\alpha} x(t)=\frac{1}{\Gamma(1-\alpha)} \int_{t_{0}}^{t}(t-\tau)^{-\alpha} x^{\prime}(\tau) d \tau .
\end{aligned}
$$

The relationship between these two definitions is given by:

$$
{ }^{C} D_{t_{0}, t}^{\alpha} x(t)={ }^{R L} D_{t_{0}, t}^{\alpha} x(t)-\frac{x\left(t_{0}\right)\left(t-t_{0}\right)^{-\alpha}}{\Gamma(1-\alpha)} .
$$

Definition 4. A continuous function $\omega:[0,+\infty) \rightarrow[0,+\infty)$ is said to belong to class- $\mathcal{K}$ if it is strictly increasing and $\omega(0)=0$, or simply call it a $\mathcal{K}$-class function.

\section{Main Results}

In this section, a fractional order Barbalat's Lemma will be introduced at first, then, some Fractional order Lyapunov-like Lemmas will be derived for fractional order nonlinear systems.

\subsection{Fractional Barbalat's lemma with Caputo operator}

Caputo fractional operator plays an important role in the fractional systems, since the initial conditions for fractional differential equations with Caputo derivatives take on the same form as for integer-order differential equations, which have well understood physical meanings. The following Barbalat's lemma is based on Caputo fractional operator.

Theorem 1. If $\int_{t_{0}}^{t} w(s) d s$ has a finite limit as $t \rightarrow+\infty$, and if ${ }^{C} D^{\alpha} w(t)$ is bounded, where $\omega:[0,+\infty) \rightarrow R$, then $w(t) \rightarrow 0$ as $t \rightarrow+\infty$, where $0<\alpha<1$. 
Proof. For proving this theorem, we will prove that $w(t)$ is uniformly continuous at first. Then, the above Fractional Barbalat's lemma will be proved through reductio ad absurdum.

Step 1. For $0 \leqslant T_{1}<T_{2}$, note that

$$
\begin{aligned}
&\left|w\left(T_{1}\right)-w\left(T_{2}\right)\right|=\frac{1}{\Gamma(\alpha)} \mid \int_{0}^{T_{1}}\left(T_{1}-s\right)^{\alpha-1}\left({ }^{C} D^{\alpha} w(s)\right) d s \\
& \quad-\int_{0}^{T_{2}}\left(T_{2}-s\right)^{\alpha-1}\left({ }^{C} D^{\alpha} w(s)\right) d s\left|=\frac{1}{\Gamma(\alpha)}\right| \int_{0}^{T_{1}}\left[\left(T_{1}-s\right)^{\alpha-1}\right. \\
&\left.\quad-\left(T_{2}-s\right)^{\alpha-1}\right]\left({ }^{C} D^{\alpha} w(s)\right) d s-\int_{T_{1}}^{T_{2}}\left(T_{2}-s\right)^{\alpha-1}\left({ }^{C} D^{\alpha} w(s)\right) d s \mid \\
& \leqslant \frac{1}{\Gamma(\alpha)}\left[\left|\int_{0}^{T_{1}}\left[\left(T_{1}-s\right)^{\alpha-1}-\left(T_{2}-s\right)^{\alpha-1}\right]\left({ }^{C} D^{\alpha} w(s)\right) d s\right|\right. \\
&\left.+\left|\int_{T_{1}}^{T_{2}}\left(T_{2}-s\right)^{\alpha-1}\left({ }^{C} D^{\alpha} w(s)\right) d s\right|\right] \\
& \leqslant \frac{M}{\Gamma(\alpha)}\left[\int_{0}^{T_{1}}\left[\left(T_{1}-s\right)^{\alpha-1}-\left(T_{2}-s\right)^{\alpha-1}\right] d s+\int_{T_{1}}^{T_{2}}\left(T_{2}-s\right)^{\alpha-1} d s\right] \\
& \leqslant \frac{M}{\Gamma(\alpha+1)}\left[T_{1}^{\alpha}-T_{2}^{\alpha}+2\left(T_{2}-T_{1}\right)^{\alpha}\right] \leqslant 2 \frac{M}{\Gamma(\alpha+1)}\left(T_{2}-T_{1}\right)^{\alpha}<\varepsilon
\end{aligned}
$$

where $\left|T_{2}-T_{1}\right|<\delta(\varepsilon)=\left(\frac{\varepsilon \Gamma(\alpha+1)}{2 M}\right)^{1 / \alpha}$. Note that $\delta$ is not depend on $T_{1}$ or $T_{2}$. According to the definition of uniformly continuous, $w(t)$ is uniformly continuous.

Step 2. Assume that $w(t)$ does not approach zero as $t \rightarrow+\infty$. Then $\exists \varepsilon_{0}>0, \forall T>0, \exists t>T,|w(t)|>\varepsilon_{0}$. Therefore, we can get an infinite sequence of $t_{i}\left(i=1,2, \ldots\right.$, and $t_{i} \rightarrow+\infty$ as $\left.i \rightarrow+\infty\right)$ such that $\left|w\left(t_{i}\right)\right|>\varepsilon_{0}$. Since $w(t)$ is uniformly continuous, $\exists \eta>0$, such that for $t^{\prime}$ and $t^{\prime \prime}$ satisfying $\left|t^{\prime}-t^{\prime \prime}\right|<\eta$, then $\left|w\left(t^{\prime}\right)-w\left(t^{\prime \prime}\right)\right|<\varepsilon_{0} / 2$, which implies that for any $t$ within the $\eta$-neighborhood of $t_{i}$, (i.e. such that $\left|t-t_{i}\right|<\eta$ ), we have $|w(t)|>\varepsilon_{0} / 2$.

Hence, for all $t_{i}$, denote $t_{0}=0$, noting that $w(s)$ keep a constant sign over the integration interval, due to the continuity of $w$ and the bounded $|w(t)|>$ $\varepsilon_{0} / 2>0$, then,

$$
\begin{aligned}
\left|\int_{t_{0}}^{t_{i}+\eta} w(s) d s\right| & =\int_{t_{0}}^{t_{i}+\eta}|w(s)| d s \\
\geqslant & {\left[\int_{t_{0}}^{t_{1}-\eta}+\int_{t_{1}-\eta}^{t_{1}+\eta}+\ldots+\int_{t_{i}-\eta}^{t_{i}+\eta}\right]|w(s)| d s \geqslant \frac{i \eta \varepsilon_{0}}{2}, }
\end{aligned}
$$

which reveals that

$$
\lim _{t \rightarrow+\infty}\left|\int_{t_{0}}^{t} w(s) d s\right|=\lim _{i \rightarrow+\infty}\left|\int_{t_{0}}^{t_{i}+\eta} w(s) d s\right|>\lim _{i \rightarrow+\infty} \frac{i \eta \varepsilon_{0}}{2} \rightarrow+\infty
$$

which contradict the known fact that $\int_{t_{0}}^{t} w(s) d s$ has a finite limit as $t \rightarrow+\infty$. So $w(t) \rightarrow 0$ as $t \rightarrow+\infty$. 
Remark 1. In [6], the convergence of a function to zero can be derived under some conditions upon its fractional integral. Noting that the function in the [6] must be bounded or has a monotonically convergent fractional order integer. However, Theorem 1 provides the result for the convergence of a function based on its fractional derivatives and integration, which is more suitable for the application in the stability analysis about fractional order systems. It is easy to find that the classical integer order Barbalat's Lemma is a special case of the above result.

Corollary 1. If $w(t)$ has a finite limit as $t \rightarrow+\infty$, and if ${ }^{C} D^{\alpha} w(t)$ is uniformly continuous. Then ${ }^{C} D^{\alpha} w(t) \rightarrow 0$ as $t \rightarrow+\infty$, where $0<\alpha<1$.

Noting that, in the analysis of the Lyapunov stability, $\mathcal{K}$-class functions are important. The following theorem is related to $\mathcal{K}$-class functions, which also is a generalization of Theorem 1 .

Theorem 2. If $\int_{t_{0}}^{t} \varphi(w(s)) d s$ has a finite limit as $t \rightarrow+\infty$, and $w(t)$ is uniformly continuous, where $w(t):[0,+\infty) \rightarrow[0,+\infty)$ and $\varphi(\cdot)$ belongs to class- $\mathcal{K}$, then $w(t) \rightarrow 0$ as $t \rightarrow+\infty$, where $0<\alpha<1$.

Proof. For proving this result, we will prove that $\lim _{t \rightarrow+\infty} \varphi(w(t))=0$ at first. Then $\lim _{t \rightarrow+\infty} w(t)=0$ can be derived based on the monotonicity of function $\varphi(\cdot)$. Assume that $\varphi(w(t))$ does not approach zero as $t \rightarrow+\infty$. Then $\exists \varepsilon_{0}>0$, $\forall T>0, \exists t>T, \varphi(w(t))>\varepsilon_{0}$. Therefore, we can get an infinite sequence of $t_{i}\left(i=1,2, \ldots\right.$, and $t_{i} \rightarrow+\infty$ as $\left.i \rightarrow+\infty\right)$ such that $\varphi\left(w\left(t_{i}\right)\right)>\varepsilon_{0}$, i.e. $w\left(t_{i}\right) \geqslant \varphi^{-1}\left(\varepsilon_{0}\right)$. Since $w(t)$ is assumed to be uniformly continuous, $\exists \eta>0$, such that for $t^{\prime}$ and $t^{\prime \prime}$ satisfying $\left|t^{\prime}-t^{\prime \prime}\right|<\eta$, then

$$
\left|w\left(t^{\prime}\right)-w\left(t^{\prime \prime}\right)\right|<\varphi^{-1}\left(\varepsilon_{0}\right) / 2,
$$

which implies that for any $t$ within the $\eta$-neighborhood of $t_{i}$, (i.e. such that $\left.\left|t-t_{i}\right|<\eta\right)$, we have

$$
|w(t)|>\varphi^{-1}\left(\varepsilon_{0}\right) / 2
$$

Hence, for all $t_{i}$,

$$
\begin{aligned}
& \left|\int_{t_{0}}^{t_{i}+\eta} \varphi(w(s)) d s\right|=\int_{t_{0}}^{t_{i}+\eta}|\varphi(w(s))| d s \\
& \quad \geqslant\left[\int_{t_{0}}^{t_{1}-\eta}+\int_{t_{1}-\eta}^{t_{1}+\eta}+\ldots+\int_{t_{i}-\eta}^{t_{i}+\eta}\right]|\varphi(w(s))| d s \geqslant \frac{i \eta \varepsilon_{0}}{2},
\end{aligned}
$$

which reveals that

$$
\lim _{t \rightarrow+\infty} \int_{t_{0}}^{t} \varphi(w(s)) d s=\lim _{i \rightarrow+\infty}\left|\int_{t_{0}}^{t_{i}+\eta} \varphi(w(s)) d s\right|>\lim _{i \rightarrow+\infty} \frac{i \eta \varepsilon_{0}}{2} \rightarrow+\infty
$$

which contradict the known fact that $\int_{t_{0}}^{t} \varphi(w(s)) d s$ has a finite limit as $t \rightarrow$ $+\infty$. So $\varphi(w(t)) \rightarrow 0$ as $t \rightarrow+\infty$, which implies $w(t) \rightarrow 0$ as $t \rightarrow+\infty$. 
Corollary 2. If $\int_{t_{0}}^{t} \varphi(w(s)) d s$ has a finite limit as $t \rightarrow+\infty$, and ${ }^{C} D^{\alpha} w(t)$ is bounded, where $w(t):[0,+\infty) \rightarrow[0,+\infty)$ and $\varphi(\cdot)$ belongs to class- $\mathcal{K}$, then $w(t) \rightarrow 0$ as $t \rightarrow+\infty$, where $0<\alpha<1$.

Proof. Due to ${ }^{C} D^{\alpha} w(t)$ is bounded, then $w(t)$ is uniformly continuous, which completes our proof.

\subsection{Fractional order Lyapunov-like lemma with Caputo operator}

The above fractional order Barbalat's lemma can be applied for studying stability of fractional order non-autonomous systems, some fractional order Lyapunov - like lemmas can be established as follows.

In what follows, we consider a general non-autonomous fractional-order nonlinear system described by Caputo derivative definition:

$$
{ }^{C} D_{t_{0}, t}^{\alpha} x(t)=f(t, x(t)),
$$

where $x(t)=\left(x_{1}(t), x_{2}(t), \ldots, x_{n}(t)\right)^{T} \in R^{n}$ and $0<\alpha<1$ denotes the state vector and fractional order of system (3.1), respectively, $f(t, x(t))=$ $\left(f_{1}\left(t, x_{1}(t)\right), f_{2}\left(t, x_{2}(t)\right), \ldots, f_{n}\left(t, x_{n}(t)\right)\right)^{T}, f_{i}\left(t, x_{i}(t)\right), i=1,2, \ldots, n$ are nonlinear continuous functions.

Theorem 3. If a scalar function $V(t, x(t))$ is positive semi-definite and the Caputo fractional derivative of $V(t, x(t))$ along the solution $x(t)$ of the system (3.1) satisfies ${ }^{C} D_{t_{0}, t}^{\alpha} V(t, x) \leqslant-\varphi(\|x(t)\|)$, where $\varphi(\cdot)$ belongs to class- $\mathcal{K}$, then $x(t) \rightarrow 0$ as $t \rightarrow+\infty$ if $x_{i}(t) i=1,2, \ldots, n$ are uniformly continuous.

Proof. Denoting that $\tilde{V}(t, x(t))=D_{t_{0}, t}^{-(1-\alpha)} V(t, x(t))$, then, based the property of Caputo's fractional-order derivative one has

$$
\dot{\tilde{V}}(t, x(t))={ }^{C} D_{t_{0}, t}^{\alpha} V(t, x) \leqslant-\varphi(\|x(t)\|) .
$$

Then, one can integrate the inequality to get $\tilde{V}(t, x(t))+\int_{t_{0}}^{t} \varphi(\|x(s)\|) d s \leqslant$ $\tilde{V}\left(t_{0}, x\left(t_{0}\right)\right)$, which implies that

$$
\lim _{t \rightarrow+\infty} \int_{t_{0}}^{t} \varphi(\|x(s)\|) d s \leqslant \tilde{V}\left(t_{0}, x\left(t_{0}\right)\right) .
$$

Noting that $x_{i}(t) i=1,2, \ldots, n$ are uniformly continuous, then, $x_{i}(t) \rightarrow 0$ as $t \rightarrow+\infty$ can be obtained according to Theorem 2 .

Corollary 3. If a scalar function $V(t, x(t))$ is is positive semi-definite and the Caputo fractional derivative of $V(t, x(t))$ along the solution $x(t)$ of Caputo system (3.1) satisfies ${ }^{C} D_{t_{0}, t}^{\alpha} V(t, x)$ is negative semi-definite, then $x(t) \rightarrow 0$ as $t \rightarrow+\infty$ if $f_{i}(t, x(t)), i=1,2, \ldots, n$ for the system (3.1) are bounded.

Proof. Since $f_{i}(t, x(t)), i=1,2, \ldots, n$ are bounded, i.e. $D_{t_{0}, t}^{\alpha} x_{i}(t)$ is bounded. Then, $x_{i}(t), i=1,2, \ldots, n$ are uniformly continuous. Thus, $x(t) \rightarrow 0$ as $t \rightarrow+\infty$ can be gotten from Theorem 3 . 
We have considered a non-autonomous case in the system (3.1), if the system (3.1) is an autonomous system, the following result can be derived:

Corollary 4. If a scalar function $V(t, x(t))$ is lower bounded and the Caputo fractional derivative of $V(x(t))$ along the solution $x(t)$ of system (3.1) ${ }^{C} D_{t_{0}, t}^{\alpha} V(x)$ is negative semi-definite, then $x(t) \rightarrow 0$ as $t \rightarrow+\infty$.

Proof. From the proof of the Theorem 3, it is easy to get $\|x(t)\|$ is bounded. As a result, $f_{i}(t, x(t)), i=1,2, \ldots, n$ are bounded due to $f_{i}\left(t, x_{i}(t)\right), i=1,2, \ldots, n$ are continuous. Thus, $x(t) \rightarrow 0$ as $t \rightarrow+\infty$ can be gotten from the above Corollary.

Remark 2. Recently, there are many results about the asymptotic stability of fractional order systems. It is easy to know that the analysis of stability in some papers can be simplified greatly by this result. For example, the proof of Theorem 1 in [26], the proof of Theorem 1 in [3].

\subsection{Fractional order Barbalat's lemma and Lyapunov-like lemmas with Riemann-Liouville operator}

Riemann-Liouville fractional operator also plays an important role in the stability analysis of fractional-order systems. According to the (2.1), one has the following results with Riemann-Liouville fractional operator.

Theorem 4. If $\int_{t_{0}}^{t} \varphi(w(s)) d s$ has a finite limit as $t \rightarrow+\infty$, and if ${ }^{R L} D^{\alpha} w(t)$ is bounded, where $w(t):[0,+\infty) \rightarrow[0,+\infty)$, then $w(t) \rightarrow 0$ as $t \rightarrow+\infty$, where $0<\alpha<1$.

Proof. By using the relationship between Riemann-Liouville's definition and Caputo's definition in (2.1) we have

$$
{ }^{C} D_{t_{0}, t}^{\alpha} w(t)={ }^{R L} D_{t_{0}, t}^{\alpha} w(t)-\frac{w\left(t_{0}\right)\left(t-t_{0}\right)^{-\alpha}}{\Gamma(1-\alpha)} .
$$

Noting that $\alpha \in(0,1)$ and $w\left(t_{0}\right) \geqslant 0$, we obtain that

$$
{ }^{C} D_{t_{0}, t}^{\alpha} w(t) \leqslant{ }^{R L} D_{t_{0}, t}^{\alpha} w(t),
$$

which implies that all conditions holds in Theorem 1. Then, it is similar to obtain $w(t) \rightarrow 0$ as $t \rightarrow+\infty$ from the proof of Theorem 1 .

Now, let us consider the following general non-autonomous fractional-order nonlinear system described by Riemann-Liouville's definition:

$$
{ }^{R L} D_{t_{0}, t}^{\alpha} x(t)=f(t, x(t))
$$

where $x(t)=\left(x_{1}(t), x_{2}(t), \ldots, x_{n}(t)\right)^{T} \in R^{n}$ and $0<\alpha<1$ denotes the state vector and fractional order of system (3.1), respectively, $f(t, x(t))=$ $\left(f_{1}\left(t, x_{1}(t)\right), f_{2}\left(t, x_{2}(t)\right), \ldots, f_{n}\left(t, x_{n}(t)\right)\right)^{T}, f_{i}\left(t, x_{i}(t)\right), i=1,2, \ldots, n$ are nonlinear continuous functions. 
Theorem 5. If a scalar function $V(t, x(t))$ is positive semi-definite and the Riemann-Liouville fractional derivative of $V(t, x(t))$ along the solution $x(t)$ of system (3.2) ${ }^{R L} D_{t_{0}, t}^{\alpha} V(t, x(t)) \leqslant-\varphi(\|x(t)\|)$, where $\varphi(\cdot)$ belongs to class- $\mathcal{K}$, then $x(t) \rightarrow 0$ as $t \rightarrow+\infty$ if $x_{i}(t), i=1,2, \ldots, n$ are uniformly continuous.

Proof. By using the relationship between Riemann-Liouville's definition and Caputo's definition in (2.1) we have

$$
{ }^{C} D_{t_{0}, t}^{\alpha} V(t, x(t))={ }^{R L} D_{t_{0}, t}^{\alpha} V(t, x(t))-\frac{V\left(t_{0}, x\left(t_{0}\right)\right)\left(t-t_{0}\right)^{-\alpha}}{\Gamma(1-\alpha)} .
$$

Noting that $\alpha \in(0,1)$ and $V\left(t_{0}, x\left(t_{0}\right)\right) \geqslant 0$, we obtain that

$$
{ }^{C} D_{t_{0}, t}^{\alpha} V(t, x(t)) \leqslant{ }^{R L} D_{t_{0}, t}^{\alpha} V(t, x(t)),
$$

which implies that all conditions holds in Theorem 3. Then, $x(t) \rightarrow 0$ as $t \rightarrow+\infty$ can be derived easily.

Corollary 5. If a scalar function $V(t, x(t))$ is positive semi-definite and the Riemann-Liouville fractional derivative of $V(t, x(t))$ along the solution $x(t)$ of Riemann-Liouville (3.2) ${ }^{R L} D_{t_{0}, t}^{\alpha} V(t, x(t)) \leqslant-\varphi(\|x(t)\|)$, where $\varphi(\cdot)$ belongs to class- $\mathcal{K}$, then $x(t) \rightarrow 0$ as $t \rightarrow+\infty$ if $f_{i}(t, x(t)), i=1,2, \ldots, n$ for the system (3.2) are bounded.

The proof is omitted here due to it is similar to the Corollary 4 .

Remark 3. There were some results about stability for the fractional order systems based on Riemann-Liouville operator [13,23, 24], noting that all of them have considered autonomous system. Compared with them, this paper has built a unified analysis method to both autonomous and non-autonomous system, which could be a improve to them.

\section{Applications}

To illustrate the result above, let us consider the asymptotic stability analysis of a simple fractional order control system. Consider the following closed-loop error dynamics for a fractional order plant with one unknown parameter is described by:

$$
D^{\alpha} e(t)=-e(t)+\sin (t) \theta(t), \quad D^{\alpha} \theta(t)=-\sin (t) e(t),
$$

where $e(t)$ is tracking error and $\theta(t)$ is parameter error. Let us analyze the asymptotic properties of this system. Consider the lower bounded function $V(t)=e^{2}(t)+\theta^{2}(t)$, then, based on some exists results [4], one has

$$
D^{\alpha} V(t) \leq 2 e(t)(-e(t)+\sin (t) \theta(t))+2 \theta(t)(-\sin (t) e(t))=-2 e^{2}(t) .
$$

Thus, according to Theorem 3 and Theorem 5 , then $e(t) \rightarrow 0$ as $t \rightarrow \infty$. Noting that just $e(t)$ converges to zero, the system is not asymptotically stable, because $\theta(t)$ is only bounded. 
Remark 4. According to the results in this paper, both Caputo's fractional order systems and Riemann-Liouville's fractional order systems could be applied. The function $V(t)$ is a zero lower bounded instead of positive definite function. The well-known Lyapunov analysis based on invariant set theorems can not be worked due to the dynamics is non-autonomous. Of course, the difficulty is still the choice of scalar function $V(t)$.

\section{Conclusions}

This paper has generalized the well-known Barbalat's Lemma to fractional order case. Both Caputo fractional operator and Riemann-Liouville fractional operator have been discussed. Then, the results have been applied to the Lyapunov stability of fractional order nonlinear systems. It's well worth exploring the applications of the proposed results to the stability analysis and control theory of delayed fractional order nonlinear system, which should be our future works.

\section{Acknowledgements}

This work was jointly supported by the Natural Science Foundation of Jiangsu Province of China under Grant No. BK20161126, the Graduate Innovation Project of Jiangsu Province under Grant No. KYLX16_0778.

\section{References}

[1] M.M. Arefi, M.R. Jahed-Motlagh and H.R. Karimi. Adaptive neural stabilizing controller for a class of mismatched uncertain nonlinear systems by state and output feedback. IEEE Transactions on Cybernetics, 45(8):1587-1596, 2015. https://doi.org/10.1109/TCYB.2014.2356414.

[2] D. Baleanu, J.A.T. Machado and A.CJ Luo. Fractional dynamics and control. Springer, Berlin Heidelberg, 2011. https://doi.org/10.1007/978-1-4614-0457-6.

[3] H. Bao, J.H. Park and J. Cao. Adaptive synchronization of fractionalorder memristor-based neural networks with time delay. Nonlinear Dynamics, 82(3):1343-1354, 2015. https://doi.org/10.1007/s11071-015-2242-7.

[4] M.A. Duarte-Mermoud, N. Aguila-Camacho, J.A. Gallegos and R. CastroLinares. Using general quadratic Lyapunov functions to prove Lyapunov uniform stability for fractional order systems. Communications in Nonlinear Science and Numerical Simulation, 22(1):650-659, 2015. https://doi.org/10.1016/j.cnsns.2014.10.008.

[5] J.A. Gallegos and M.A. Duarte-Mermoud. Boundedness and convergence on fractional order systems. Journal of Computational and Applied Mathematics, 296(1):815-826, 2016. https://doi.org/10.1016/j.cam.2015.11.008.

[6] J.A. Gallegos, M.A. Duarte-Mermoud, N. Aguila-Camacho and R. CastroLinares. On fractional extensions of Barbalat lemma. Systems \& Control Letters, 84(1):7-12, 2015. https://doi.org/10.1016/j.sysconle.2015.07.004. 
[7] M. Hou, G. Duan and M. Guo. New versions of Barbalats lemma with applications. Journal of Control Theory and Applications, 8(4):545-547, 2010. https://doi.org/10.1007/s11768-010-8178-z.

[8] J.B. Hu, G.P. Lu, S.B. Zhang and L.D. Zhao. Lyapunov stability theorem about fractional system without and with delay. Communications in Nonlinear Science and Numerical Simulation, 20(3):905-913, 2015. https://doi.org/10.1016/j.cnsns.2014.05.013.

[9] H.B. Jiang. Hybrid adaptive and impulsive synchronisation of uncertain complex dynamical networks by the generalised Barbalat's lemma. Control Theory \& Applications IET, 3(10):1330-1340, 2009. https://doi.org/10.1049/ietcta.2008.0335.

[10] C. Kou, Y. Yan and J. Liu. Stability analysis for fractional differential equations and their applications in the models of HIV-1 infection. Computer Modeling in Engineering and Sciences, 39(3):301-318, 2009.

[11] N. Laskin. Fractional market dynamics. Physica A: Statistical Mechanics and its Applications, 287(3-4):482-492, 2000. https://doi.org/10.1016/S03784371(00)00387-3.

[12] K. Li, W. Yu and Y. Ding. Successive lag synchronization on nonlinear dynamical networks via linear feedback control. Nonlinear Dynamics, 80(1):421-430, 2015. https://doi.org/10.1007/s11071-014-1879-y.

[13] R. Li and W. Chen. Lyapunov-based fractional-order controller design to synchronize a class of fractional-order chaotic systems. Nonlinear Dynamics, 76(1):785-795, 2014. https://doi.org/10.1007/s11071-013-1169-0.

[14] Y. Li, Y.Q. Chen and I. Podlubny. Mittag-Leffler stability of fractional order nonlinear dynamic systems. Automatica, 45(8):1965-1969, 2009. https://doi.org/10.1016/j.automatica.2009.04.003.

[15] M. Liu. Optimal harvesting policy of a stochastic predatorcprey model with time delay. Applied Mathematics Letters, 48(1):102-108, 2015. https://doi.org/10.1016/j.aml.2014.10.007.

[16] S. Liu, W. Jiang, X. Li and X.F. Zhou. Lyapunov stability analysis of fractional nonlinear systems. Applied Mathematics Letters, 51(1):13-19, 2016. https://doi.org/10.1016/j.aml.2015.06.018.

[17] R. Magin. Fractional calculus in bioengineering. Begell House Publishers, Redding, 2006.

[18] R.L. Magin. Fractional calculus models of complex dynamics in biological tissues. Computers \& Mathematics with Applications, 59(5):1586-1593, 2010. https://doi.org/10.1016/j.camwa.2009.08.039.

[19] A.E. Matouk and A.A. Elsadany. Achieving synchronization between the fractional-order hyperchaotic Novel and Chen systems via a new nonlinear control technique. Applied Mathematics Letters, 29(1):30-35, 2014. https://doi.org/10.1016/j.aml.2013.10.010.

[20] R. Metzler and J. Klafter. The random walk's guide to anomalous diffusion: a fractional dynamics approach. Physics reports, 339(1):1-77, 2000. https://doi.org/10.1016/S0370-1573(00)00070-3.

[21] N. Shimizu and W. Zhang. Fractional calculus approach to dynamic problems of viscoelastic materials. JSME International Journal Series C, 42(4):825-837, 1999. https://doi.org/10.1299/jsmec.42.825. 
[22] J. Slotine and W. Li. Applied nonlinear control. Englewood Cliffs, NJ: PrenticeHall, 1991.

[23] F. Wang, Y. Yang and M. Hu. Asymptotic stability of delayed fractional-order neural networks with impulsive effects. Neurocomputing, 154(22):239-244, 2015. https://doi.org/10.1016/j.neucom.2014.11.068.

[24] F. Wang, Y. Yang, M. Hu and X. Xu. Projective cluster synchronization of fractional-order coupled-delay complex network via adaptive pinning control. Physica A: Statistical Mechanics and its Applications, 434:134-143, 2015. https://doi.org/10.1016/j.physa.2015.03.089.

[25] Z. Wu, Y. Xia and X. Xie. Stochastic Barbalat's lemma and its applications. IEEE Transactions on Automatic Control, 57(6):1537-1543, 2012. https://doi.org/10.1109/TAC.2011.2175071.

[26] J. Yu, C. Hu, H. Jiang and X. Fan. Projective synchronization for fractional neural networks. Neural Networks, 49(1):87-95, 2014. https://doi.org/10.1016/j.neunet.2013.10.002.

[27] R. Zhang and S. Yang. Robust synchronization of two different fractional-order chaotic systems with unknown parameters using adaptive sliding mode approach. Nonlinear Dynamics, 71(1):269-278, 2013. https://doi.org/10.1007/s11071-0120659-9. 